\title{
El periodismo móvil y la creación de contenidos informativos en Internet. El caso de la revista digital Living Palermo
}


El periodismo móvil se está convirtiendo en un nuevo nicho de mercado. La creación de contenidos y la proliferación de las app específicas para smartphone otorga mucho más protagonismo a este tipo de dispositivos. Cuando hablamos de periodismo móvil el éxito está conectado a algunas de las características que comparte con los teléfonos móviles, como la portabilidad, la ubicuidad o la utilización personal. Pero también está relacionada con las capacidades propias de estos mismos modelos, como la multimedialidad, la hipertextualidad y la interactividad.

La siguiente propuesta tiene como objetivo demostrar la viabilidad del periodismo móvil a partir de la experiencia de Living Palermo, una revista digital elaborada únicamente con un dispositivo móvil. Mediante el análisis del desarrollo de una revista digital a partir de un smartphone o tableta se examinará la viabilidad del periodismo móvil como modus operandi para la creación de contenidos periodísticos en Internet y si esto cumple con los estándares de calidad mínimos para poder ser un publicación digital rentable. Con esto, también se busca detectar las habilidades y herramientas que un profesional de la comunicación necesita a la hora de gestionar los contenidos y distribuirlos. La figura del periodista polivalente supone un papel esencial para poder desarrollar este tipo de proyectos.

El estudio del caso demuestra, pese a algunas limitaciones, que se puede realizar una publicación digital que cumpla unos mínimos cualitativos periodísticos mediante la generación de contenido, única y exclusivamente, a través de una herramienta móvil.

Palabras clave: periodismo móvil, multimedia, smartphone, interactividad, publicación digital.

\section{Introducción}

El periodismo móvil se está convirtiendo en un nuevo nicho de mercado, ya que la creación de contenidos y la proliferación de las app específicas para smartphone otorgan mucho más protagonismo a este tipo de dispositivos.

Cuando hablamos de periodismo móvil, el éxito de los smartphones está conectado a algunas de las características que comparte con los teléfonos móviles, como la portabilidad, la ubicuidad o la utilización personal. Pero también está relacionada con las capacidades propias de estos mismos modelos, como la multimedialidad, la hipertextualidad y la interactividad facilitada por los altos niveles de usabilidad (Canavilhas, 2013).

Las posibilidades que se abren a los contenidos periodísticos vía móvil son inmensas. La consolidación del carácter multimedia de los 
contenidos en el móvil revolucionará la manera de contar historias; la velocidad y la movilidad de la conexión a Internet, consolidan, por otro lado, al teléfono como puerta de acceso a la Red (Cebrián Herreros y Flores Vivar, 2011: 69).

La posibilidad de testear la viabilidad de realizar periodismo mediante un teléfono móvil, de forma que se respeten los estándares de calidad $y$ se cumplan las características de multimedialidad, hipertextualidad e interactividad. Crear contenidos de todo tipo, ya sea videos, podcasts o artículos mediante un Smartphone, acentúa, más si cabe, la tarea un periodista polivalente. Del periodista que solo se centra en elaborar una pieza informativa se está pasando cada vez más al profesional que también se ocupa de grabar los cortes de voz o efectuar las fotografías del suceso. Los dispositivos móviles tipo smartphones y tabletas añaden un elemento nuevo, el de la movilidad, con suficiente capacidad para transformar las competencias de los profesionales del periodismo (González Molina, 2013).

La capacidad para crear contenidos con un mínimo de calidad periodística requiere el cumplimiento de siete características del webperiodismo identificadas por diferentes autores (Bardoel y Deuze, 2000; Díaz Noci, 2001; Machado y Palacios, 2013; Salaverría, 2005; Zamith, 2008).

- Hipertextualidad: posibilidad de conectar documentos digitales o diferentes partes de un documento a través de enlaces.

- Multimedialidad: combinación integrada de texto, imagen y sonido.

- Interactividad: capacidad de permitir al usuario interactuar con los contenidos.

- Personalización: capacidad de ofrecer al usuario la información específica para su interés.

- Perennidad/memoria: capacidad de almacenar y organizar información de forma permanente para consulta.

- Instantaneidad/actualización continua: capacidad de añadir nuevas informaciones de inmediato.

- Ubicuidad: capacidad de acceder a la información en cualquier momento y desde diferentes puntos.

En la discusión de los resultados se pretende averiguar si estas característica son identificables en las aplicaciones para iPhone, y buscar alguna eventual conexión entre los contenidos para Web y para smartphones (Canavilhas, 2009: 11).

Se busca detectar qué habilidades y capacidades necesita el profesional del periodismo para conseguir información, distribuir contenidos y relacionarse con la audiencia. El auge que actualmente experimenta el mercado de dispositivos móviles aconseja el abordaje académico de esta cuestión, máxime cuando la comunicación vive un 
momento convulso con los medios, en el que se replantea su modelo de negocio (Casero-Ripollés, 2010).

Actualmente, los informadores desarrollan todo tipo de funciones dentro de la redacción de sus medios, desde grabar y elaborar sus propias piezas informativas en les televisiones, hasta redactar para la versión impresa y digital. Se han vuelto polivalentes. Una situación que la actual crisis económica acentúa, ya que las empresas apuestan por la reducción de costes y la optimización de los recursos, desembocando en denominaciones como la del periodista orquesta para aludir a esta realidad (González i Ortells, 2012). En Living Palermo se ha desarrollado este papel de periodista orquesta.

Con este nuevo panorama comunicativo, Living Palermo ha sido una apuesta en la que la multimedialidad y la inmediatez han supuesto dos requisitos indispensables para realizar la publicación. La explotación de estas posibilidades ha sido fundamental para crear un producto de calidad. Una forma de realizar periodismo en vivo y en directo para demostrar que el periodismo móvil es posible ante este nuevo marco mediático en el que los actores comunicativos cada vez son más.

\section{Objetivos}

El primer objetivo es desarrollar una publicación digital especializada en el calendario social y cultural del barrio de Palermo en Buenos Aires, la revista digital Living Palermo. La utilización de una herramienta móvil para desarrollar una plataforma óptima para este tipo de acción y la generación de contenido propio son clave para cumplir este primer objetivo. Sin el cumplimiento de este no se podría establecer el segundo: valorar la viabilidad del periodismo móvil como modus operandi en la publicación digital. Al tratarse del estudio de un caso concreto en el que se ha llevado a cabo un proyecto práctico, Living Palermo, se realizará un análisis a través de la metodología con la que se ha desarrollado el proyecto.

\section{Material y método}

Living Palermo es una publicación digital llevada a la práctica durante el desarrollo de un Trabajo de Final de Grado del Grado en Periodismo. De este modo, el estudio del caso propuesto y sus resultados se desarrollarán en el siguiente epígrafe, se detallarán los resultados y cada una de las fases. 
Al tratarse de un estudio de caso, cabe destacar las diferentes fases que se han tenido en cuenta y los procedimientos para desarrollar toda la publicación digital; los resultados. En primer lugar, la fase de producción, donde se realizó un estudio comparativo y un análisis de la competencia.

\section{Preproducción}

Para empezar a diseñar Living Palermo, era necesario estudiar los modelos ya existentes para delimitar con exactitud la temática y la forma de la publicación digital. Para ello se realizó un trabajo de análisis de la competencia de los distintos medios de comunicación, tanto privados como públicos, que ofrecieran información sobre Palermo, ya fuera de actualidad como social o cultural.

En primer lugar, el Gobierno de Buenos Aires tiene una página web en la que se puede encontrar información de carácter cultural, una especie de programa donde se ofrece toda la información acerca de los eventos organizados por el Gobierno de la Capital Federal. No está exclusivamente dedicada a esta labor, ya que en ella también se ofrece información de servicio para el ciudadano, así como para los turistas que desean visitar la ciudad, por lo tanto, podríamos decir que no se trata de una página web que puramente trata el contenido de carácter social y cultural en el que se centrará la publicación digital que se desarrolla.

En segundo lugar, Agenda Cultural de Buenos Aires es una página web que también depende del Gobierno de Buenos Aires, en este caso se puede seleccionar la oferta cultural por barrios. También posee un perfil en Twitter y Facebook. Sin embargo, como la página web anterior, no realizan ningún tipo de análisis, reportaje, crónica... Tampoco tiene ningún apartado multimedia dedicado a describir cómo ha sido el acontecimiento marcado en la agenda cultural. En este caso, el poder incluir reportajes, ya sean multimedia, escritos o radiofónicos, permite ampliar las posibilidades y realizar un tipo de servicio diferente.

Palermo Online es un portal de noticias de este barrio porteño. Su diseño está bastante obsoleto, no está actualizado a las últimas novedades de Internet. Publica de tres a cuatro noticias relacionadas con el barrio por día, pero en ningún momento hay signos de multimedialidad, interactividad o hipertextualidad. Es un volcado de las notas de prensa que emiten los diferentes colectivos públicos o privados.

En este caso, lo que diferencia a Living Palermo de la competencia podría describirse en de la siguiente forma: además de actuar como una agenda cultural, también incluye y habla de la actualidad del barrio, no se centra tan solo en los acontecimientos culturales sino también sociales.

En segundo lugar, dentro de la fase de preproducción se centra también la selección de contenidos. El objetivo de Living Palermo era realizar, al menos, tres publicaciones semanales, ya que la actividad cultural es extensa y existen muchos temas de creación propia. Ha sido una apuesta arriesgada, ya que es necesario tenerlo muy bien 
programado para poder llevar la publicación digital al día. Se delimitaron los días para poder compatibilizar este trabajo con los días de asistencia a clase. La distribución de los contenidos para cada día se ha ido realizando poco a poco para poder cuadrar todas las fechas.

Había un total de 21 publicaciones programadas, que finalmente han pasado a ser 39 (número total de post de la publicación) desde el miércoles 1 de abril hasta el lunes 25 de mayo, debido a la gran actividad social y cultural del barrio. La selección de contenidos ha sido precisa y concisa, se ha intentado en todo momento elaborar piezas que tuvieran cabida en la publicación y que fueran de interés periodístico.

La selección de contenidos ha sido muy variada, existen entradas que hablan sobre política pero también hay apartados dedicados a la naturaleza o el fútbol. También cabe destacar que cada una de las entradas, y dependiendo de su contenido, ha recibido un tratamiento distinto. Más tarde detallaré esto en el apartado de producción.

A continuación, se adjunta la tabla con el calendario de publicaciones completamente detallado, poco a poco y durante el proceso de elaboración esta selección de contenidos ha ido modificándose. La actualidad primaba por encima de todo, de modo que algunos temas que en un principio había programados finalmente no han tenido cabida en la publicación.

ABRIL

\begin{tabular}{|c|c|c|c|c|c|c|}
\hline LUNES & MARTES & MIÉRCOLES & JUEVES & VIERNES & SÁBADO & DOMINGO \\
\hline 30 & 31 & $\begin{array}{l}1 \text { Inicio } \\
\text { publicación }\end{array}$ & 2 & 3 & 4 & 5 \\
\hline $\begin{array}{l}6 \\
\text { Un paseo } \\
\text { por los } \\
\text { bosques de } \\
\text { Palermo }\end{array}$ & 7 & 8 & $\begin{array}{l}9 \\
\text { Un partido } \\
\text { en el } \\
\text { Monumental }\end{array}$ & 10 & 11 & $\begin{array}{l}12 \\
\text { Los } \\
\text { resultados } \\
\text { del paro } \\
\text { de } \\
\text { transporte }\end{array}$ \\
\hline 13 & $\begin{array}{l}14 \\
\text { Feria } \\
\text { Gastronómica } \\
\text { Le Marché }\end{array}$ & 15 & 16 & $\begin{array}{l}17 \\
\text { BAFICI }\end{array}$ & 18 & $\begin{array}{l}19 \\
\text { Feria del } \\
\text { libro } \\
\text { permanent } \\
\text { e }\end{array}$ \\
\hline $\begin{array}{l}20 \\
\mathrm{ECOBICl}\end{array}$ & 21 & 22 & $\begin{array}{l}23 \\
\text { Elecciones, } \\
\text { ¿cómo se } \\
\text { organizan en } \\
\text { Buenos } \\
\text { Aires? }\end{array}$ & 24 & 25 & $\begin{array}{l}26 \\
\text { Cubrimient } \\
\text { o } \\
\text { elecciones } \\
\text { en directo }\end{array}$ \\
\hline $\begin{array}{l}27 \\
\text { Nota post- } \\
\text { elecciones }\end{array}$ & $\begin{array}{l}28 \\
\text { Nota feria } \\
\text { libro }\end{array}$ & $\begin{array}{l}29 \\
\text { Galería } \\
\text { Feria del } \\
\text { Libro }\end{array}$ & $\begin{array}{l}30 \\
\text { Nota Turf 1을 } \\
\text { Mayo }\end{array}$ & $\begin{array}{l}1 \\
\text { Entrevista } \\
\text { feria libro }\end{array}$ & 2 & $\begin{array}{l}3 \\
\text { Visita } \\
\text { Jardín } \\
\text { Botánico } \\
\end{array}$ \\
\hline
\end{tabular}


MAYO

\begin{tabular}{|c|c|c|c|c|c|c|}
\hline LUNES & MARTES & MIÉRCOLES & JUEVES & VIERNES & SÁBADO & $\begin{array}{l}\text { DOMING } \\
0\end{array}$ \\
\hline $\begin{array}{l}4 \\
\text { Hipódromo } \\
\text { Palermo }\end{array}$ & 5 & 6 & 7 & $\begin{array}{l}8 \\
\text { Reportaje } \\
\text { radiofónico } \\
\text { "Little Italy" }\end{array}$ & $\begin{array}{l}9 \\
\text { Visita } \\
\text { MALBA }\end{array}$ & 10 \\
\hline 11 & $\begin{array}{l}12 \\
\text { Datos Feria } \\
\text { del Libro } \\
2015\end{array}$ & 13 & $\begin{array}{l}14 \\
\text { No } \\
\text { comment } \\
\text { Feria del } \\
\text { Libro }\end{array}$ & $\begin{array}{l}15 \\
\text { Reportaje } \\
\text { Creatavist } \\
\text { Feria del } \\
\text { Libro }\end{array}$ & 16 & 17 \\
\hline 18 & 19 & 20 & 21 & 22 & 23 & 24 \\
\hline $\begin{array}{l}25 \\
\text { Fin publicación }\end{array}$ & 26 & 27 & 28 & & 30 & 31 \\
\hline 1 & 2 & 3 & 4 & 5 & 6 & 7 \\
\hline
\end{tabular}

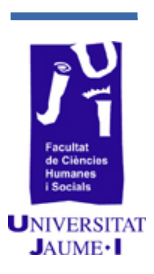

La previsión de los gastos en cuestión de material y de programas para edición ha sido escasa. En primer lugar, como instrumento imprescindible para poder desarrollar este proyecto. El smartphone ha sido la pieza fundamental, concretamente el iPhone 6 equipado con los programas de edición para imagen, video y audio. Todo eso no ha tenido un coste cero, ya que tenía el material antes de empezar esta publicación. El programa de edición de video iMovie, incorporado en el iPhone 6; el programa de edición de imagen PicLab, descargado gratuitamente desde el programa de app para iPhone; el programa de edición de audio Audacity, de descarga gratuita para cualquier soporte; la plataforma Wordpress, con la que he desarrollado toda la publicación, de utilización gratuita, y por último la agenda, para ello se utilizaron $2,99 €$ en la aplicación iCalendar, para sincronizarla con Google Calendar y poder desarrollar la agenda en la publicación digital.

\section{Producción}

En cuanto a la fase de producción, para empezar con la publicación, después de haber establecido algunos esbozos en la fase de preproducción, la plataforma se definió con el nombre de Living Palermo. Un nombre que definía a la perfección este proyecto, en castellano "Viviendo Palermo». Acompañado de un subtítulo: «una ventana a la vida social del barrio», que acotaba lo que se podía esperar de esta publicación, la temática y a qué estaba dedicada.

Una vez elegido el nombre se creó una identidad corporativa que fuera sencilla y que invitara al lector a entrar en esta plataforma y consultar los contenidos. Mediante la aplicación PicLab se creó el logotipo que ha sido desde un primer momento la imagen de Living Palermo, escrito en letras blancas y acompañado de un color azul claro, que se 
instauró en todas las plataformas que he utilizado para publicar cualquier tipo de contenido. Esa ha sido la seña de identidad.

Para empezar con el diseño y darle forma, se utilizó la plataforma Wordpress, ya que, además de haberla utilizado en algunas asignaturas de la universidad, creo que es muy completa para un tipo de publicación digital como Living Palermo. Tiene numerosas posibilidades para enlazar contenido multimedia $\mathrm{y}$ hacer del proyecto un lugar interactivo $\mathrm{y}$ atractivo para el potencial lector.

Además de contar con Wordpress, también se utilizaron dos redes sociales a través de las que se han viralizado los contenidos, como son Twitter e Instagram, que también forman parte del diseño de Living Palermo. Con Twitter, no solo se ha viralizado contenido, sino que se ha creado contenido propio, que más tarde en el apartado de interactividad se explicará.

Esta publicación ha estado hecha y pensada para utilizarse a través de un dispositivo móvil, el proyecto ha sido desde un principio crear contenido con el teléfono móvil para que cualquier persona a través de su celular pudiera consultarlo, de manera que se eligió una plantilla en Wordpress que fuera lo más visual posible, que se adaptara a las condiciones de un smartphone y que a la vez diera la posibilidad de acceder desde la computadora.

Para poder dividir el contenido de manera adecuada y situar los contenidos de la mejor forma posible se estableció un menú con nueve secciones diferentes:

- Actualidad: En esta sección se incluyen las principales noticias y reportajes ligados con la actualidad más pura del barrio, las principales novedades o acontecimientos que se celebren en Palermo.

- Calendario de actividades: mediante la aplicación iCalendar y Google Calendar, se ha incluido un calendario en la publicación digital en la que los lectores pueden observar cuándo y dónde tendrán lugar los principales eventos en el barrio de Palermo.

- ¿Qué hacer en Palermo?: una sección creada para aquellos que deseen visitar el barrio de Palermo, es decir, en ella se incluirán los lugares que visitar. Es un apartado enfocado a los turistas.

- Los mejores lugares de Palermo: un recorrido por los principales lugares de Palermo, en él se incluyen fotografías y reportajes de los lugares más representativos de este barrio.

- Galería: en la galería tiene cabida todo tipo de fotografías sobre las diferentes temáticas de la publicación digital.

- Multimedia: además de las fotografías, también se incluyen videos y locuciones con temáticas diferentes, así como distintos contenidos realizados con soportes especializados para un tema determinador, en este caso, Creatavist o Prezi. 
- ¿Qué es Living Palermo?: qué es esta publicación digital y cuáles son sus objetivos, su enfoque y el público al cual va dirigido.

- ¿Quién soy?: una breve descripción de quién soy y de qué hago en Buenos Aires realizando esta publicación digital como Trabajo de Final de Grado.

- Contacto: un apartado dedicado a aquellos que deseen ponerse en contacto con Living Palermo.

El menú de inicio es muy visual, en la parte superior izquierda aparece el logotipo y a continuación se pueden encontrar las entradas, todas representadas mediante una fotografía y un título y organizadas por orden de publicación, de más reciente a menos. La barra de menú se encuentra arriba a la derecha, hay que desplegarla para poder ver cómo se dividen los contenidos, hay un total de nueve secciones que en el apartado siguiente explicaremos.

Además, para dar a conocer las redes sociales, en la parte inferior de la publicación se encuentran enlazados el Twitter e Instagram de la publicación. Asimismo la agenda se encuentra en dos lugares: en una de las barras del menú y en la parte inferior junto con las dos redes sociales utilizadas.

La interactividad de la página se ha conseguido a través de diferentes herramientas. En primer lugar, las redes sociales, que han sido Twitter e Instagram, a pesar de no tener numerosos seguidores, siempre que se ha publicado alguna entrada en Living Palermo el contenido se ha adaptado mediante enlaces y fotografías en cada una de estas plataformas. Además, con Twitter, se han realizado coberturas en directo de diversos eventos, como han sido las elecciones Primarias, Abiertas, Simultáneas y Obligatorias de la Ciudad de Buenos Aires (PASO) y de la 41. a Feria Internacional del Libro de Buenos Aires. Mediante esta propuesta, se conseguía relatar en directo mediante 140 caracteres, videos e imágenes, lo que estaba sucediendo, sacándole el máximo partido a la posibilidad de hacer periodismo móvil. Esta idea surgió a partir de la visualización del Twitter de Carmela Ríos, periodista en Unidad Editorial y que explora nuevas narrativas en dispositivos móviles y redes sociales. Su visión y su forma de narrar fue relevante a la hora relatar los hechos de esta forma.

Los enlaces e hipervínculos en cada uno de los artículos que se han publicado en Living Palermo también han propiciado esta interactividad y navegación por capas. En algunos artículos se ha redirigido al lector a diferentes enlaces dentro de la misma publicación digital, como ha sido en el caso de que una galería complementara directamente al reportaje realizado de manera escrita.

Los lectores también contaban con una zona donde podían dejar cualquier comentario y establecer así comunicación e interactividad con la publicación. Cada una de las entradas de Living Palermo, contaba con etiquetas para posicionar la página web en el buscador de Google. 
Otro de los puntos que cabe destacar en el apartado de interactividad es la cantidad de plataformas que se han utilizado para hacer la publicación más amena. Google Maps ha ayudado a localizar en todas la publicaciones, a designar de manera visual el lugar en el que se encuentra situado el lugar del que se habla durante una publicación, ha sido una herramienta fundamental. Otra de las que se han utilizado ha sido Creatavist, para desarrollar un reportaje multimedia y explotar así todas las cualidades de la publicación, volcando imagen, texto, audio y video en una misma entrada. Y por último Prezi, con la que se han plasmado datos de manera distinta para que el lector interactuara de manera diferente con los números.

Dejando de lado esto, hablando ahora de la interactividad que se ha producido entre los usuarios, se ha analizado la pequeña muestra de visitas durante los 55 días de publicación, con un total de 1165.

Para poder empezar este proyecto se tuvieron que destinar las dos primeras semanas de la estancia en Buenos Aires a conocer el barrio. Se desconocía por completo la ciudad y Palermo, por lo tanto se hizo un trabajo de investigación en el que se intentó conocer, en primer lugar, los sitios más atractivos y se fue profundizando, más tarde, en cuestiones de actualidad. No fue una tarea fácil, ya que el barrio de Palermo es uno de los más grandes de Buenos Aires en extensión y con más habitantes, alrededor de 250.000. Además, es el centro neurálgico de la mayoría de los acontecimientos que se celebran. También cuenta con muchos centros culturales y parques al aire libre, lo cual lo convierte en un gran atractivo para turistas y residentes en la ciudad porteña.

Una vez explorado Palermo se comenzaron las labores de diseño de la publicación y de imagen, esto llevó dos semanas más. Perfeccionar la página, crear un perfil en las redes sociales y conseguir una homogeneidad en la imagen de Living Palermo para poder empezar a publicar.

Durante este tiempo también se hizo una agenda de temas sobre los que se podía trabajar, la mayoría de ellos atemporales y sin periodicidad. Por este motivo, se tuvo que modificar en numerosas ocasiones el calendario de publicaciones, ya que la actualidad es lo que prima y a medida que pasaba el tiempo acontecían hechos relevantes que tenían cabida y eran atractivos para la publicación.

El día 1 de abril se inauguró Living Palermo, una vez con las ideas claras y definidas. El camino hasta llegar aquí no fue fácil, pero todo lo que ha seguido tampoco. El desconocimiento de las fuentes y de la geografía ha sido en ocasiones una problemática. Pero, finalmente, en cada una de las publicaciones que se han realizado han conseguido un estándar de calidad para el artículo, ya fuere a través de Internet o acercándome a la fuente directamente.

El elevado ritmo de publicaciones, no menos de tres a la semana, ha supuesto más de un mes y medio de dedicación plena a Living Palermo. Para cada uno de los temas, se trató de contactar con las fuentes que 
pudieran aportar la información adecuada para realizar cualquiera de los géneros que he desarrollado en la publicación.

\section{Postproducción}

Y por último la fase de postproducción, donde el smartphone tiene un papel fundamental.

Todas las fotografías de Living Palermo han sido tomadas con el iPhone 6 y las que han requerido edición han sido retocadas con la aplicación PicLab. El reportaje televisivo y los videos han sido íntegramente grabados y editados con el iPhone 6, con el programa iMovie. El sonido también he podido editarlo mediante este programa. Los audios subidos a la plataforma Ivoox también se han grabado y editado con el iPhone 6 y con el programa Audacity.

\section{Discusión y conclusiones}

El desarrollo de esta publicación digital ha puesto de manifiesto que es posible realizar contenido periodístico de calidad a través de un dispositivo móvil. La ventaja de poder elaborar el contenido al momento, editarlo, subirlo a la plataforma y divulgarlo por las diferentes redes sociales, hace que el periodismo digital cumpla con una de sus máximas actuales: la inmediatez. Además, la interactividad que se ofrece a partir de los enlaces y la viralización de contenidos, promulgan una lectura multimedia y por capas que favorece la explotación del medio.

Los smartphones son cada vez más plataformas de entretenimiento y dominadas por la presencia de información. Obviamente, existen diferencias entre el periodismo móvil y la web, pero las limitaciones, como la dimensión de la pantalla o el incorrecto funcionamiento de la red en algunas localizaciones, son insignificantes frente al nuevo mundo de posibilidades que se abre con el gran potencial que ofrecen hoy en día los smartphones en cuanto a creación de contenido para una plataforma online.

\section{Bibliografía}

Artículos en la web:

BUTLER, P. La velocidad y el contexto, factores claves para una estrategia de periodismo móvil. Disponible en: https://ijnet.org/es/blog/lavelocidad-y-el-contexto-factores-claves-para-una-estrategia-deperiodismo-m\%C3\%B3vil [Última consulta: 7 abril, 2015].

Gómez Escalonilla, G. Periodismo digital: nuevas exigencias para el profesional de la información. Disponible en: http://www.compoliticas.org/redes/pdf/redes1/7pdf [Consulta: 7 abril, 2015]. 
Artículos en publicaciones periódicas

CANAVILHAS, J. (2009): Contenidos informativos para móviles: estudio de aplicaciones para iPhone. Universidad da Beira, Portugal.

- (2013): «El periodismo en los tiempos de un nuevo ecosistema mediático: propuestas para la enseñanza superior», Universidad da Beira, Portugal.

Canavilhas, J. y otros (2013): Hacia el periodismo móvil. Portal de Comunicaciones Iberoamericanas, Santiago de Chile.

CASERo Ripollés, A. (2010): "Prensa en Internet: nuevos modelos de negocio en el escenario de la convergencia», El profesional de la información.

Cebrián Herreros, M. y J. M. Flores Vivar (2011): Periodismo en la telefonía móvil. Madrid.

González Molina, S. y S. ORtells Badenes (2008): «La polivalencia periodística de los profesionales en redes», Estudios sobre el mensaje periodístico. 